\section{IUMRS General Assembly Meets in Boston}

The General Assembly of the International Union of Materials Research Societies met December 1 in Boston during the MRS Fall Meeting. Delegates to the Assembly reported on activities of the adhering bodies they represented and discussed upcoming IUMRS-sponsored events and plans. Reports were given by MRS-T (Taiwan), C-MRS (China), MRS-K (Korea), MRS-J (Japan), MRS (United States), A-MRS (Australia), M-MRS (Mexico), and E-MRS (Europe). MRS-I (India) was not represented.

\section{IUMRS-Sponsored Meetings}

Li-Chung Lee reviewed plans for the International Conference on Electronic Materials (IUMRS-ICEM-94), December 19-22 in Hsinchu, Taiwan. The meeting incorporates 10 symposia covering ULSI, sensors, compound semiconductors, packaging display materials, superconductors, recording media and other topics. See the March 1994 MRS Bulletin, p. 59, for an update.

The 1994 International Conference in Asia (IUMRS-ICA-94) will be held in Taiwan immediately after IUMRS-ICEM94. The 1994 meeting will emphasize structural materials, e.g., advanced ceramics, composite materials, intermetallics, structural polymers, and advanced processing. MRS-T President Li-Chung Lee is serving as conference chair and Chien-Min Wang is chair of the program committee. See the March 1994 MRS Bulletin, p. 57, for more information.

Gabriella Diaz summarized preliminary plans for the 1995 International Conference on Advanced Materials (IUMRS-ICAM-95). It will be hosted by Mexican-MRS August 27-September 1, 1995 at the convention center in Cancún, Mexico. Diaz introduced a preliminary list of 25 symposia topics. The conference chairs will be M. José Yacamán, Instituto de Fisica, UNAM, Mexico and R. Chianelli, Exxon Research and Engineering, USA.

\section{IUMRS News}

Li-Chung Lee (MRS-T) was nominated and elected unanimously to be treasurer of IUMRS for 1994 and 1995. The position of secretary general was created, and R.P.H. Chang, past president of IUMRS, agreed to serve in this position.

Shigeyuki Somiya reported on the six Young Scientist Awards presented at the ICAM Meeting in Tokyo, September 1993 (see the December 1993 MRS Bulletin, p. 62). These awards will also be given at subsequent ICEM and ICAM meetings.

A model for IUMRS membership was adopted. Individual MRS societies may be formed and join IUMRS, but their application must be endorsed by previously existing MRS societies in their geographical area. This is meant to facilitate communication among MRS societies in neighboring regions and to encourage formation of larger, well-integrated MRS societies. After two years of legal existence, a recognized MRS organization can be presented to enter IUMRS by a geographic neighbor.

\section{Adhering Body Activities}

MRS-T president Li-Chung Lee reported on plans for IUMRS-ICEM-94 and IUMRS-ICA-94 to be held in Taiwan.

C-MRS President, Heng-De Li, reported that the membership of C-MRS has grown from 500 to 1200 in the past year. $\mathrm{Li}$ reported that C-MRS hosted IUMRSICA-93, September 6-10, 1993, immediately following IUMRS-ICAM-93, on a boat on the Yangtze River, and that it drew about 160 attendees. The annual CMRS conference, which focused on phase transformations and solidification, was held November 16-19, 1993 in Beijing. It drew 138 attendees. In 1993 C-MRS began publising the bimonthly Chinese Journal of Materials Science and Technology. The Chinese Journal of Materials Research (formerly Progress of Materials Science) was scheduled to begin publication in 1994.

Min Che Chon, president of MRS-K, announced that the 1993 MRS-K Fall Meeting was held November 19-20, 1993 at Yonsei University in Seoul, drawing 300 attendees. The 2nd Joint MRSMexico/MRS-Korea Symposium on advanced materials was scheduled for December 6-10, 1993 in Monterrey, Mexico. MRS-K's bimonthly publication, Korean Journal of Materials Research, has a current circulation of about 900 , with topics covering $30 \%$ metals, $10 \%$ ceramics, $14 \%$ polymers, and $45 \%$ electronic materials.

MRS-J activities, as reported by 1993 president Masao Doyama, included the MRS-J 5th Annual Meeting December 9 and 10, 1993 at Kanagawa Science Park, featuring symposia on organic and inorganic hybrid materials, biomedical materials, and advanced materials. The summer meeting is scheduled for July 21 and 22, 1994 in Kawasaki, Japan. MRS-J will cooperate with Japanese societies and institutions (AESJ, CRIEPI, JAERI, and
PNC) in hosting the XVIII International Symposium on the Scientific Basis for Nuclear Waste Management in Kyoto, Japan, October 23-27, 1994. (No similar symposium will be held during the MRS Fall Meeting this year.) See the March 1994 MRS Bulletin, p. 59 for more information. MRS-J is now affiliated with Materials Letters.

1993 MRS president Tom Picraux reported on the 1993 MRS Spring and Fall Meetings, with attendances of 2,400 and about 3,700 , respectively. As of the end of October, membership had reached 11,600, an increase of 5\% over 1992.

Jim S. Williams (A-MRS) recapped the Ion Beam Modification meeting held in February 1993. He also spoke about the Compound Optoelectronic Materials and Devices (COMAD) meeting scheduled for December 1993. The Ninth International Conference on Ion Beam Modification of Materials is scheduled for February 5-10, 1995 in Canberra, Australia.

Gabriella Diaz, representing M-MRS, reported on the Materials Issues in Art and Archaeology symposium planned for May 16-20, 1994 in Cancún. The meeting is being organized in cooperation with MRS and will explore the interface between traditional materials science, the history of technology, and the archaeological and conservation sciences. See the March 1994 MRS Bulletin, p. 56, for more information.

Ian Boyd, representing E-MRS, reported on the 4 th European East/West Conference and Exhibition and the 1993 E-MRS Fall Meeting held October 17-21 in St. Petersburg, Russia. Seven symposia drew 730 attendees, 650 of them from Eastern nations. The E-MRS Spring Meeting will be May 24-27, 1994 in Strasbourg, France. For more information about the meeting, see the January and March 1994 issues of the MRS Bulletin, p. 58 and 36 , respectively.

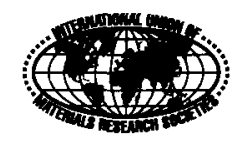

News about IUMRS conferences and other activities should be sent to: MRS Bulletin, Materials Research Society, 9800 McKnight Road, Pittsburgh, PA 15237-6006. Fax: (412) 367-4373. 


\section{ATTENTON}

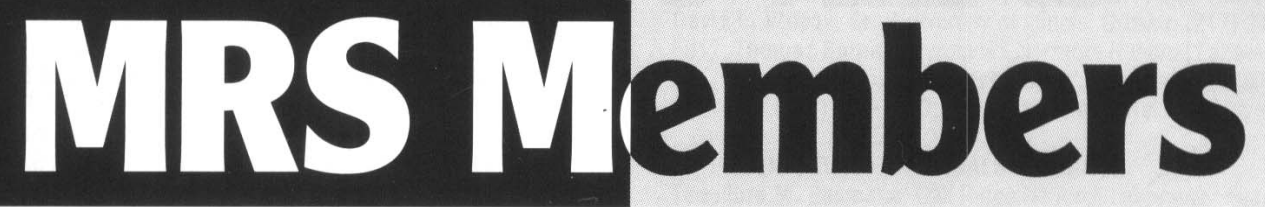

\section{Please update your address for the 1994 Membership Directory}

(Publication date: August 1)

1. Check the address label on your copy of the MRS Bulletin.

2. Fill in the form below if you want a different address to be included in the Membership Directory.

\section{Deadline for Directory Listings is May 13, 1994}

To USA Members: New postal automation requires a street address or P.O. Box validated by the U.S. Postal Service. (This is true even for universities.) If you do not know your street address or P.O. Box, please contact your mailroom or your local postmaster.

My Membership Directory address should read as follows:

Name ID\#

Institution Dept.

(on mailing label)

Address Check if home $\square$ business $\square$

VERY IMPORTANT: Please include valid Street Address or P.O. Box

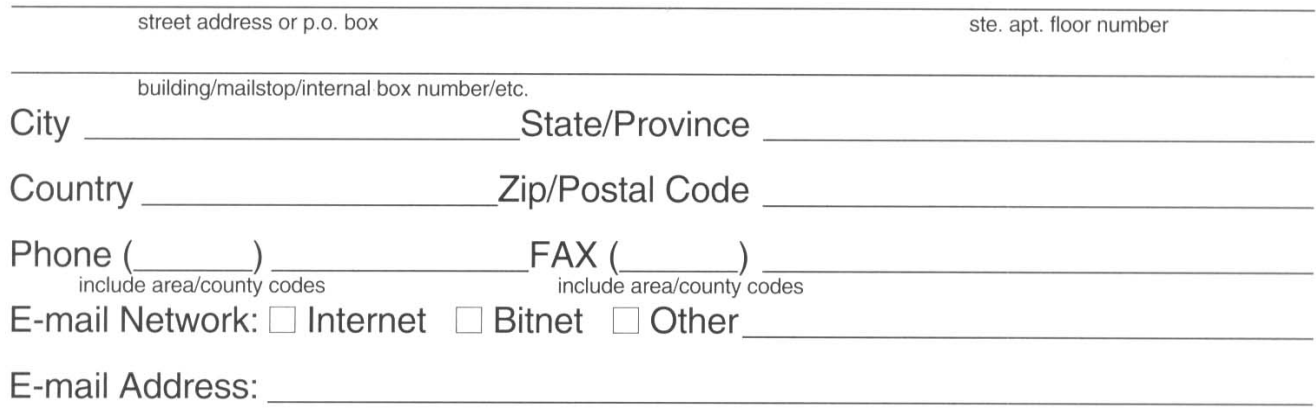

$\square$ Please send my mail to the address above.

$\square$ Please send my mail to a different address, as follows: $\nabla$

My address for mailings should read as follows:

Institution Dept.

Address Check if home $\square$ business $\square$

VERY IMPORTANT: Please include valid Street Address or P.O. Box

\begin{tabular}{lr}
\hline street address or p.o. box & ste. apt. floor number \\
\hline City $\quad$ building/mailstop/internal box number/etc. & State/Province \\
Country & Zip/Postal Code
\end{tabular}




\section{CALL FOR PAPERS}

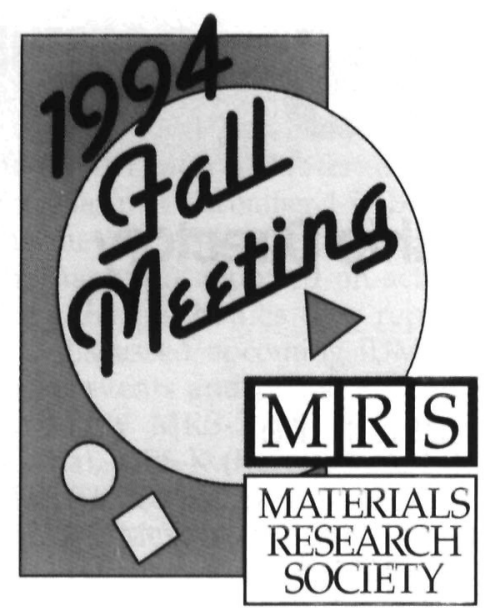

November 28 - December 2, 1994

Boston Marriott Hotel and

Westin Hotel/Copley Place

Sheraton Boston Hotel

Boston, Massachusetts

\section{Abstract Deadline: June 20, 1994 SYMPOSIA}

\section{A: BEAM-SOLID INTERACTIONS FOR MATERIALS SYNTHESIS AND}

\section{CHARACTERIZATION}

David E. Luzzi, University of Pennsylvania, (215) 898-8366. Fax (215) 573-2128 luzzi@sol1.Irsm.upenn.edu; Masaya Iwaki, The Institute of Physical and Chemical Research, Japan, (81) 48-462-1111. Fax (81) 48-462-4623, iwaki@rkna50. riken.go.jp; Tony F. Heinz, IBM T.J. Watson Research Center. (914) 945-2333, Fax (914) 9453715, heinz@watson.ibm.com; Dale C. Jacobson, AT\&T Bell Laboratories, (908) 5826557; Fax (908) 582-4228, dcj@physics.att.com

\section{B1: EVOLUTION OF THIN-FILM AND SURFACE STRUCTURE AND MORPHOLOGY} B.G. Demczyk. The University of Michigan. (313) 936-3353, Fax (313) 763-5567, temcom@caen.engin.umich.edu; Eric Garfunkel, Rutgers University, (908) 932-2747, Fax (908) 932-5312, gar@@chemb.rutgers.edu; Ellen D. Williams, The University of Maryland, (301) 405-6156. Fax (301) 314-9465, williams@surface.umd edu: Bruce M. Clemens, Stanford University, (415) 725-7455, Fax (415) 725-4034, clemens@sierra.stanford.edu; J.E. Cuomo, North Carolina State University, (919) 515-7218, Fax (919) 515-7724, cuomo@mat.mte.ncsu.edu

\section{B2: IHIN FILMS: STRESSES AND MECHANICAL PROPERTIES V}

Shefiord P. Baker, Max-Planck-Institut für Metallforschung, Germany, (49) 711 2095-214, Fax (49) 711-2095-250, baker@vaxww1.mpi-stuttgart.mpg de; Paul H. Townsend, Dow Chemical Company. (517) 636-8120, Fax (517) 636-6558, townsendph@dow.com; Peter Børgesen, Cornell University, (607) 255-4045 Fax (607) 255-6575, borg@msc.cornell.edu; Caroline A. Ross, Komag, Inc., (408) 957-4400, Fax (408) 263-9449, caross@leland.stanford edu; Cynthia A. Volkert, AT\&T Bell Laboratories, (908) 582-3001, Fax (908) 582-4228, thia@physics.att.com

\section{C: STRUCTURE AND PROPERTIES OF INTERFACES IN CERAMICS}

Dawn A. Bonnell, The University of Pennsylvania, (215) 898-6231, Fax (215) 573-2128; Uma Chowdhry, E.I. DuPont de Nemours \& Co. Inc., (302) 774-5721, Fax (302) 773-6213; Manfred Rühle, Max-Planck-Institut für Metallforschung, Germany, (49) 711-2095-319, Fax (49) 711-2095-295

\section{D: ATOMIC-LEVEL CONTROL OF EPITAXIAL HETEROSTRUCTURES}

P.I. Cohen, University of Minnesota, (612) 625-5517, Fax (612) 625-4583, cohen@ ee.umn.edu; B.G. Orr, University of Michigan, (313) 936-3609, Fax (313) 764-2193, orr@server.physics.Isa.umich.edu; C.J. Palmstrom, Bellcore, (908) 758-2934, Fax (908) 758-4372. palms@nyquist.cc.bellcore.com; J.D. Tersoff, IBM T.J. Watson Research Center, (914) 945-3138, Fax (914) 945-2141, tersoff@watson.ibm.com

\section{E: CHEMICAL PERSPECTIVES OF MICROELECTRONICS MATERIALS}

Vincent M. Donnelly, AT\&T Bell Laboratories, (908) 582-3471, Fax (908) 582-3427 Wayne L. Gladfelter. University of Minnesota, (612) 624-4391, Fax (612) 626-7541 Stephen M. Gates, IBM T.J. Watson Research Center, (914) 945-2665, Fax (914) 945-2141; Jennifer J. Zinck, Hughes Research Laboratories, (310) 317-5913 Fax (310) $317-5450$

\section{F: MICROCRYSTALLINE AND NANOCRYSTALLINE SEMICONDUCTORS}

Louis Brus, AT\&T Bell Laboratories, (908) 582-3119, Fax (908) 582-3958; Robert W. Collins, The Pennsylvania State University, (814) 865-3059, Fax (814) 865-2326 Masataka Hirose, Hiroshima University, Japan, (81) 824-24-7655, Fax (81) 824-227038; Frederick Koch, Technische Universität Münich, Germany, (49) 89-3209-2342, Fax (49) 89-3209-2317; Chuang Chuang Tsai, Xerox PARC, (415) 812-4515, Fax (415) 493-6349

\section{G: SCIENCE AND TECHNOLOGY OF FULLERENE MATERIALS}

Patrick Bernier Université de Montpellier. France, (33) 6714-3538, Fax (33) 67522504; Donald S. Bethune, IBM Almaden Research Center, (408) 927-2480, Fax (408) 927-2100; Long Y. Chiang, National Taiwan University, Taiwan, (886) 2-362-5507, Fax (same as phone); Thomas W. Ebbesen, NEC Corporation, Japan, (81) 298-566107, Fax (81) 298-56-6136; Robert A. Metzger, University of Alabama, (205) 3485952, Fax (205) 348-9104; John W. Mintmire, Naval Research Laboratory, (202) 767-2026, Fax (202) 767-3321

\section{H: HIGH T, SUPERCONDUCTIVITY - MATERIALS AND APPLICATIONS}

David S. Ginley, National Renewable Energy Lab, (303) 231-7873, Fax (303) 231 1381; Jurgen Halbritter, Kernforschungszentrum Karlsruhe, IMF, Germany, (49) 7247-823398, Fax (49) 7247-824567; Jon S. Martens, Conductus, Inc., (408) 5249818, Fax (408) 737-6699; Michael P. Siegal, Sandia National Laboratories, (505) 845-9453. Fax (505) 844-5459; Tomoji Kawai, Osaka University, Japan, (81) 6-8775111, Ext. 3552, Fax (81) 6-875-2440

\section{1: MATERIALS FOR SMART SYSTEMS}

Susan Trolier-McKinstry, The Pennsylvania State University, (814) 863-8348, Fax (814) 865-2326, stm1@alpha.mrl.psu.edu; Marilyn Wun-Fogle, Naval Surface Warfare Center, (301) 394-3648, Fax (301) 394-3018 or 394-3499, marilyn@ chaos.nswc.navy.mil; Kenji Uchino, The Pennsylvania State University, (814) 8638035, Fax (814) 865-2326; Easo P. George, Oak Ridge National Laboratory, (615) 574-5085, Fax (615) 574-7659; Sadayuki Takahashi, NEC Corporation, Japan, (81) 44-856-2164, Fax (81) 44-856-2128

\section{2: FERROELECTRIC THIN FILMS IV}

Seshu B. Desu, Virginia Tech, (703) 231-6820, Fax (703) 231-8919; R. Ramesh, Bellcore, (908) 758-3126, Fax (908) 758-4372; Bruce A. Tuttle, Sandia National Laboratories, (505) 845-8026, Fax (505) 844-2974; T. Shiosaki, Kyoto University, Japan, (81) 75-753-5309, Fax (81) 75-753-5749

\section{Ja: ENGINEERING OF NANOSTRUCTURED MATERIALS}

Jackie Y. Ying, Massachusetts Institute of Technology, (617) 253-2899, Fax (617) 258-8224; Merrilea J. Mayo. The Pennsylvania State University, (814) 863-7330. Fax (814) 865-2917; Yet-Ming Chiang, Massachusetts Institute of Technology, (617) 253-6471, Fax (617) 253-6201: Lawrence T. Kabacoff, Office of Naval Research, (703) 696-0283, Fax (703) 696-0934

\section{Jb: GRAIN-SIZE AND MECHANICAL PROPERTIES-FUNDAMENTALS AND APPLICATIONS}

N.J. Grant, Massachusetts Institute of Technology, (617) 253-5637, Fax (617) 258-8836; M.A. Otooni. U.S. Army, (201) 724-5746, Fax (201) 361-7378; R.W. Armstrong, University of Maryland, (301) 405-5291, Fax (301) 314-9477; T.N. Baker, University of Strathclyde, United Kingdom, Kozo Ishizaki, Nagaoka University of Technology, Japan, (81) 258-47-3067, Fax (81) 258-46-6972, ishizaki@voscc. nagaokaut.ac.jp

K: CHEMICAL VAPOR DEPOSITION OF REFRACTORY METALS AND CERAMICS III Woo Y. Lee, Oak Ridge National Laboratory, (615) 576-2894, Fax (615) 574-6918, wyu@ornl.gov; Bernard M. Gallois, Stevens Institute of Technology, (201) 216-5263, Fax (201) 216-8306; Michael A. Pickering, Morton International, (617) 937-9103, Fax (617) 933-5142

\section{L: HIGH-TEMPERATURE ORDERED INTERMETALLIC ALLOYS-VI} Joe Horton, Oak Ridge National Laboratory, (615) 574-5575, Fax (615) 574-7659, htn@ornl.gov; Ian Baker, Dartmouth College, (603) 646-2184, Fax (603) 646-3856 ian.baker@dartmouth.edu; Shuji Hanada, Tohoku University, Japan, (81) 22-2152115, Fax (81) 22-215-2116; Ronald D. Noebe, NASA Lewis Research Center, (216) 433-2093, Fax (216) 433-8011; Dan Schwartz, McDonnell Douglas Aerospace, (314) 232-6835, Fax (314) 232-0888

\section{M: CERAMIC MATRIX COMPOSITES-ADVANCED HIGH-TEMPERATURE} STRUCTURAL MATERIALS

Richard A. Lowden, Oak Ridge National Laboratory, (615) 576-2769, Fax (615) 5768424; Mattison K. Ferber, Oak Ridge National Laboratory, (615) 576-0818, Fax (615) 574-4913; John R. Hellmann, The Pennsylvania State University, (814) 865-0163, Fax (814) 863-4718; Steven G. DiPietro, Textron Specialty Materials, (508) 4545488, Fax (508) 934-7593; Krishan K. Chawla, New Mexico Institute of Mining and Technology, (505) 835-5229, Fax (505) 835-5626 


\section{N: DYNAMICS IN SMALL CONFINING SYSTEMS}

J.M. Drake, Exxon Research and Engineering Co., (908) 730-2848, Fax (908) 7303042; J. Klafter, Tel Aviv University, Israel, (972) 3-6408254, Fax (972) 3-6409293; S.M. Troian, Princeton University, (609) 258-4574, Fax (609) 258-0211; R. Kopelman, University of Michigan, (313) 764-7541, Fax (313) 747-4865

\section{Oa: COMPUTATIONAL APPROACHES AND APPLICATIONS TO PREDICTING} PROPERTIES OF COMPLEX MATERIALS

James R. Chelikowsky, University of Minnesota, (612) 625-4837, Fax (612) 6267246, jrc@msi.umn.edu; Steven G. Louie, University of California, Berkeley, (510) 642-1709, Fax (510) 643-9473, louie@jungle.berkeley.edu; John Northrup, Xerox PARC, (415) 812-4117, Fax (415) 812-4140, john_northrup.parc@xerox.com; Eric Shirley, Lawrence Livermore National Laboratory, (510) 423-4667, Fax (510) 422-2851, shirley@cabernet.IInl.gov

Ob: APPLICATIONS OF INNOVATIVE KNOWLEDGE BASES IN MATERIALS DESIGN Al Jackson, WL/MLIM, (513) 255-8787, Fax (513) 476-7995; jacksoag@mlgate.ml. af.mil; Kosuke Ishii, Ohio State University, (614) 292-8486, Fax (614) 292-3163, ishii.2@osu.edu; Steve LeClair, WL/MLIM, (513) 255-8787, Fax (513) 476-7995, leclaisr@mlgate.ml.af.mil; C.L. Philip Chen, Wright State University, (513) 873-5107, Fax (513) 873-5133, pchen@kiwi.cs.wright.edu

\section{P: FRACTAL ASPECTS OF MATERIALS}

Fereydoon Family, Emory University, (404) 727-4293, Fax (404) 727-0873, phytf@emory.edu; Paul Meakin, University of Oslo, Norway, (47) 22-856415, Fax (47) 22-855101, meakin@lys.uio.no; Bernard Sapoval, EcoléPolytechnique, France, (33) 169-334-172, Fax (33) 169-333-004, bs@pmcsunl.polytechnique.fr; Richard Wool, University of Illinois, (217) 333-2468, Fax (217) 333-27363, r-wool@ux1.cso.uiuc.edu

\section{Q: CHARACTERIZATION AND PROPERTIES OF DEFECTS IN POLYMER MATERIALS}

David C. Martin, University of Michigan, (313) 936-3161, Fax (313) 763-4788, Dave_Martin@mse.engin.umich.edu; Christopher Viney, University of Washington, (206) 543-7450, Fax (206) 543-3100, cviney@u.washington.edu; Ronald G. Larson, AT\&T Bell Laboratories, (908) 582-6158, Fax (908) 582-5570, rgl@likewise.att.com

\section{R: POLYMER MATRIX COMPOSITES}

Albert F. Yee, University of Michigan, (313) 763-2565 or 764-4312, Fax (313) 7636010 , albert_yee@mse.engin.umich.edu; Lynn S. Penn, University of Kentucky, (606) 257-7897, Fax (606) 258-1929, penn@engr.uky.edu; Shaw Ming Lee, Ciba-Geigy Corporation, (714) 779-9000, Ext. 203, Fax (714) 777-0628; Steve Granick, University of Illinois, Urbana-Champaign, (217) 333-5720, Fax (217) 333-2736, sgranick@uiuc.edu

\section{S: BIOMOLECULAR AND BIOMIMETIC MATERIALS}

Paul Calvert, University of Arizona, (602) 322-2994, Fax (602) 322-2993; Joe Cesarano, Sandia National Laboratories, (505) 272-7624, Fax (505) 272-7304; Robert Marchessault, McGill University, Canada, (514) 398-6276, Fax (514) 3987249; Jitka Solc, Dow Chemical Company, (517) 636-1890, Fax (517) 636-6558, Ina Goldberg, Allied Signal Inc., (201) 455-3684, Fax (201) 455-4239

\section{T: SYNTHESIS AND PROPERTIES OF ADVANCED CATALYTIC MATERIALS}

Enrique Iglesia, University of California-Berkeley, (510) 642-9673, Fax (510) 642 4778, iglesia@ns144.cchem.berkeley.edu; Dick Nagaki, Union Carbide Corporation, (304) 747-5940, Fax (304) 747-5430, dan@medinah.atc.ucarb.com; Peter Lednor Shell Research B.V., The Netherlands, (31) 20-630-3571, Fax (31) 20-630-4038, lednor1@ksla.nl; Levi Thompson, University of Michigan, (313) 936-2015, Fax (313) 763-0459, Itt@engin.umich.edu;

\section{U: SOLID STATE IONICS}

Gholam-Abbas Nazri, GM NAO R\&D Center, (313) 986-0737, Fax (313) 986-2244 Jean-Marie Tarascon, Bell Communication Research, (908) 758-2930, Fax (908) 758-4372, Martha Schreiber, Daimler-Benz AG, Germany, (49) 731-505-2210, Fax (49) 731-505-4208

\section{Va: MICROSTRUCTURE OF CEMENT-BASED SYSTEMS}

Sidney Diamond, Purdue University, (317) 494-5016, Fax (317) 496-1364, diamond@ecn.purdue.edu; Fred P. Glasser, Aberdeen University, United Kingdom, (44) 22-427-2906, Fax (44) 22-427-2921; Lillian D. Wakeley, U.S. Army Waterways Experiment Station, (601) 634-3215, Fax (601) 634-3242

\section{Vb: BONDING AND INTERFACES IN CEMENTITIOUS MATERIALS}

Sidney Mindess, University of British Columbia, Canada, (604) 822-2899, Fax (604) 822-7006; Jan Skalny, Canada, (416) 932-9531, Fax (416) 480-1367; Larry Roberts, W.R. Grace \& Co.-Conn., (617) 498-4823, Fax (617) 498-4313

\section{W1: ADVANCES IN POROUS MATERIALS}

Sridhar Komarneni, The Pennsylvania State University, (814) 865-1542, Fax (814) 865-2326; Douglas M. Smith, University of New Mexico, (505) 277-2861, Fax (505) 277-1024; Jeffrey S. Beck, Mobil Research \& Development Corporation, (609) 7374017, Fax (609) 737-5217
W2: HOLLOW AND SOLID SPHERES AND MICROSPHERES - SCIENCE AND TECHNOLOGY ASSOCIATED WITH THEIR FABRICATION AND APPLICATION Morris Berg, University of Illinois, Urbana, (217) 333-1457, Fax (217) 244-6917; David L. Wilcox, Sr., University of Illinois, Urbana, (217) 244-2808, Fax (217) 244 6917; Thomas Bernat, Lawrence Livermore National Laboratory, (510) 422-5915, Fax (510) 422-4982; Joe K. Cochran, Jr., Georgia Institute of Technology, (404) 894 2851, Fax (404) 853-9140; David Kellerman, Kellerman Associates, (508) 486-0053

\section{$\mathrm{X}$ : FRONTIERS OF MATERIALS RESEARCH}

Theodore M. Besmann, Oak Ridge National Laboratory, (615) 574-6852, Fax (615) 574-6918, tmb@ornl.gov; Timathy D. Sands, University of California, Berkeley. (510) 642-2347, Fax (510) 643-5792, sands@uclink berkeley.edu; Gary S. Was, University of Michigan, (313) 763-4675, Fax (313) 763-4540, gary_was@um.cc.umich.edu

\section{Y: MICROSTRUCTURE OF IRRADIATED MATERIALS}

Ian M. Robertson, University of Illinois, Urbana, (217) 333-6776, Fax (217) 3332736; Lynn E. Rehn, Argonne National Laboratory, (708) 252-5021, Fax (708) 252 4798; Steven J. Zinkle, Oak Ridge National Laboratory, (615) 576-7220, Fax (615) 574-0641; Will J. Phythian, AEA Technology, United Kingdom, (44) 235-43-4342, Fax (44) 235-43-5941

\section{Za: OPTICAL WAVEGUIDE MATERIALS}

Alice E. White, AT\&T Bell Laboratories, (908) 582-2506, Fax (908) 582-2783 aew@allwise att com; Dennis G. Hall, University of Rochester, (716) 275-2134. Fax (716) 273-1072, hall@moe.optics.rochester.edu; Douglas W. Hall, Corning. Inc. (607) 974-3602, Fax (607) 974-3675, Hiroo Kanamori, Sumitomo Electric Industries, Ltd., Japan, (81) 45-853-7166, Fax (81) 45-851-1565

\section{Zb: MATERIALS FOR OPTICAL LIMITING}

Robert Crane, WL/MLPJ, (513) 255-3808, Ext. 174, Fax (5i3) 255-1128, craner|@ ml.wpafb af.mil; Eric Van Stryland, University of Central Florida, (407) 658-6814 Fax (407) 658-6880, ewvs@admin.creol.ucf.edu; Keith Lewis, Defense Research Agency, United Kingdom, (44) 684-895062, Fax (44) 684-894540; Monte Khoshnevisan, Rockwell Science Center, (805) 373-4271, Fax (805) 373-4775 mkhoshne@scimail.remnet.rockwell.com

\section{AA: APPLICATIONS OF SYNCHROTRON RADIATION TECHNIQUES TO MATERIALS SCIENCE}

Dale L. Perry, Lawrence Berkeley Laboratory, (510) 486-4819, Fax (510) 486-5799 Neal Shinn, Sandia National I aboratory, (505) 844-5457, Fax (505) 844-5470. Kevin D'Amico, X-Ray Analytics, Ltd., (516) 282-2065, Fax (516) 282-5239: Gene Ice, Oak Ridge National Laboratory, (615) 574-2744, Fax (615) 574-7659; Louis Terminello, Lawrence Livermore National Laboratory, (510) 423-7956. Fax (510) 423-7040

\section{BB: NEUTRON SCATTERING IN MATERIALS SCIENCE}

Dan A. Neumann, NIST, (301) 975-5252. Fax (301) 921-9847; Thomas P Russell IBM Almaden Research Center, (408) 927-1638, Fax (408) 927-3310; Bernhardt J. Wuensch, Massachusetts Institute of Technology, (617) 253-6889, Fax (617) 2586478

\section{CC: MATERIALS SCIENCE AND HIGH-MAGNETIC FIELDS}

Laurence Campbell, Los Alamos National Laboratory, (505) 667-1482. Fax (505) 665-4311; Reza Abbaschian, University of Florida, (904) 392-6609, Fax (904) 392 6359; Donald U. Gubser, Naval Research Laboratory, (202) 767-2926, Fax (202) 404 8009; T.P. Smith III, IBM T.J. Watson Research Center, (914) 945-2809, Fax (914) $945-1484$

For general meeting information, contact:

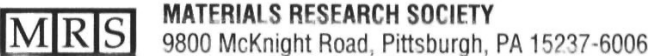 (412) 367-3003, Fax (412) 367-4373}

\section{MEETING CHAIRS}

Theodore M. Besmann, MS 6063, Metals and Ceramics Division, Oak Ridge National Laboratory, P. 0. Box 2008, Oak Ridge, TN 37831-6063, (615) 574-6852 Fax: (615) 574-6918, tmb@ornl.gov

Timothy D. Sands, Department of Materials Science and Mineral Engineering University of California, Berkeley, Hearst Mining Building, Berkeley, CA 94720 (510) 642-2347, Fax (510) 643-5792, sands@uclink.berkeley.edu

Gary S. Was, Departments of Nuclear Engineering and Materials Science and Engineering, University of Michigan, 213 Cooley Building, Ann Arbor, MI 48109 (313) 763-4675, Fax (313) 763-4540, gary was@um.cc.umich.edu 